\title{
Transferring Cultural Geo-History Datasets of Artifacts Using GIS Geodatabase for Archiving in Dodoma Capital City, Tanzania
}

\author{
Augustino Mwakipesile ${ }^{1}$, Edwin Ngowi ${ }^{2}, \mathrm{Ng}^{\prime}$ winamila Kasongi ${ }^{1}$, Emmanuel Temu ${ }^{3}$ \& Rehema Kilonzo ${ }^{2}$ \\ ${ }^{1}$ Department of Geography and Environmental Studies, The University of Dodoma, Dodoma, Tanzania \\ ${ }^{2}$ Department of Development Studies, The University of Dodoma, Dodoma, Tanzania \\ ${ }^{3}$ Department of History, The University of Dodoma, Dodoma, Tanzania \\ Correspondence: Edwin Ngowi, Department of Development Studies, The University of Dodoma, P.O. Box 395, \\ Dodoma, Tanzania. E-mail: ngowiee@yahoo.com; ngowiee@udom.ac.tz
}

Received: February 4, 2018

doi:10.5539/jsd.v11n2p88
Accepted: February 22, $2018 \quad$ Online Published: March 30, 2018

URL: https://doi.org/10.5539/jsd.v11n2p88

\begin{abstract}
The study aimed to create a historical geographic information system (HGIS), including a geodatabase and simple web application for Dodoma Capital City (DCC) in Tanzania. A web GIS application based is an outcome of the study that can improves research on, and knowledge of, the rare artifacts of cultural and historical heritage in Dodoma Capital City (DCC) for historians and the wider academic community. Likewise, spatial data incorporated allows for visualization of the relationship between people, and their geographic and cultural surroundings. Therefore, the cultural geo-history in this paper describes the specific connection of the cultural artifacts and historical site in a given area to their environment and geographic space. For that purpose, the Dodoma Capital City (DCC) historical artifacts as a case study were cataloged based on GIS techniques, geocoding protocols, and describing the artifacts to create an intuitive and familiar tool for historical researchers and archivists to better understand the cultural geo-history of Dodoma Capital City (DCC). The resulting tool, the Dodoma Capital City Historical Geographical Information System (DCCHGIS), combines a geodatabase and a web application to provide access to a small portion of the geospatial cultural history of Dodoma Capital City (DCC). The DCCHGIS demonstrates that archiving are useful in creating an accurate, informative, and usable Historical Geographic Information System (HGIS) tool that increase the knowledge of and access to cultural geo-history.
\end{abstract}

Keywords: cultural geo-history, artifacts, Geodatabase, historical geographic information system

\section{Introduction}

In the year 1973, Government of United Republic of Tanzania (URT) designated Dodoma Municipal to become the Capital City of Tanzania (URT, 1976; URT, 2014). The urbanization rate between 1973 and 2005 was mute despite being selected by popular referendum as Tanzania's new capital in 1973. Low urbanization rate is explained by failure to follow through that decision, with the majority of government departments remaining in Dar es Salaam (Wenban-Smith, 2014). It is also unfortunately the case that Dodoma has few particular advantages, apart from a central location (Ibid). Contributing factors to low urbanization may be a result of debates over misconception on perceived harsh environmental conditions, perceived inadequate socio-economic services to host influx of migrants and government budget constraints. However, between 1988 and 2012, urbanization rate has increased tremendously (Table 1).

On migration, the internal migration by place of residence in 2011 indicated that some of the regions that experienced net migration loss by place of birth data experienced positive net migration by current migration data such as Dodoma (URT, 2015).

According 1967 census (Table 2), Dodoma municipal had about 23,559 human population, whereas, 2012 census reported 213,636 human population with a projection of tremendous population increase following Government's decision to ensure within four years from July 2016 all core Government activities, including Ministries are moved to Dodoma. 
Table 1. Dodoma urbanization and urban growth

\begin{tabular}{ccccccc}
\hline \multicolumn{2}{l}{ Urbanization (\%) } \\
\hline 1978 & 1988 & 2002 & 2012 & $1978-1988$ & $1988-2002$ & $2002-2012$ \\
8.8 & 10.6 & 12.6 & 15.4 & 4.4 & 3.5 & 4.2 \\
\hline
\end{tabular}

Source: Wenban-Smith, 2014; Tanzania Bureau of Statistics, 2016

Table 2. Evolution of Dodoma Capital population $1967-2012$

\begin{tabular}{ccccccccc}
\hline \multicolumn{1}{l}{ Population } \\
\hline 1967 & 1978 & 1988 & 2002 & 2012 & $67-78$ & $78-88$ & $88-02$ & $02-12$ \\
23559 & 45807 & 83205 & 150604 & 410956 & 6.23 & 6.15 & 4.33 & 6.3 \\
\hline
\end{tabular}

Source: Wenban-Smith, 2014; Tanzania Bureau of Statistics, 2016

To cope with high urbanization rate and planning the capital city in a best way, the Government of URT through Capital City Development Authority (CDA) and Dodoma Municipal Council (DMC) have managed to plan and allocate land for different uses according to Capital City Master Plans of 1976, 1988 and 2010 respectively. These efforts have attained to make the Capital City the way it is today and further, foresees its growth in a best possible way unlike other cities in developing countries. However, conservation of the capital city artifacts has not been done up to the expectation. It is even astonishing, the Tanzania Sustainable Cities Programme Environmental and Social Impact Assessment (ESIA) overlooked adverse impacts on artifacts and historical sites that may be associated with urbanization outcomes (URT, 2010). As a result, there is a great likelihood of loosing ample history and culture for education, research, tourism and other recreational purposes if no prompt actions are put forward to rectify the situation. The main focus of this study was to create a historical GIS database and online GIS web application for rare artifacts for preservation of cultural and historical heritage of Dodoma Capital City (DCC). This is due to the fact that the most widely used content standard for archival collections in Tanzania is the describing archives, personal papers, and manuscripts standard, however, this standard do not accommodate the availability of new electronic sources and protocols for digital collections. Therefore, Mapping and Conservation of Dodoma Capital City Artifacts and Historical Sites study provides geographic visualization, allows basic spatial data, presents visual representation of the photographs, and establishes user friendly geographic reference protocols for archival data. This purpose completed using the tools available with ArcGIS Desktop program and linked with developed ArcGIS Online website and application options. As such, this study documents geocoding protocols and methods in order to establish a reusable process in creating HGIS using archival and historical data while providing a case study in the DCCHGIS itself.

\section{Materials and Methods}

\subsection{The Study Area}

The study areas encompass Dodoma urban district and Chamwino district council. Purposely, selection of these councils rest on the facts of high urbanization rate following decision by the government of Tanzania to move all government activities to Dodoma by 2020. This move is likely to cause negative impacts and so calling for a timely study to identify and map artifacts in the area.

\subsection{Data Collection Methods}

The methods used to create the Dodoma Capital City Historical Geographic Information System (DCCHGIS) merge procedures from artifacts/archival processing with skills and techniques from geographic information science. The $\mathrm{X}$ and $\mathrm{Y}$ coordinates (location) of the artifacts and historical site were collected by using Garmin 64S GPS device and data were projected into WGS 84 Datum. For non-spatial data, a well designed questionnaire was used to collect descriptive information of the sampled artifacts and historical site. The Geodatabase creation was carried out based on the steps proposed by Reeve (1996): need analysis, logical design, physical design and testing, implementation and maintenance. Moreover, dimension measurements (such as height, length and width) of artifacts and historical site was carried out to ensure adequate and proper documentation. Practical considerations in establishing Geodatabase structure and data validity helped to create a straightforward and easy-to-use base for the entry of data collected using standard archival collection processing procedures. Figure 2 outlines the workflow that was followed while creating the Historic Geographic 
Information System (HGIS). In the following sections, each phase is described in greater detail in order to allow for reproduction of the database and web application creation.

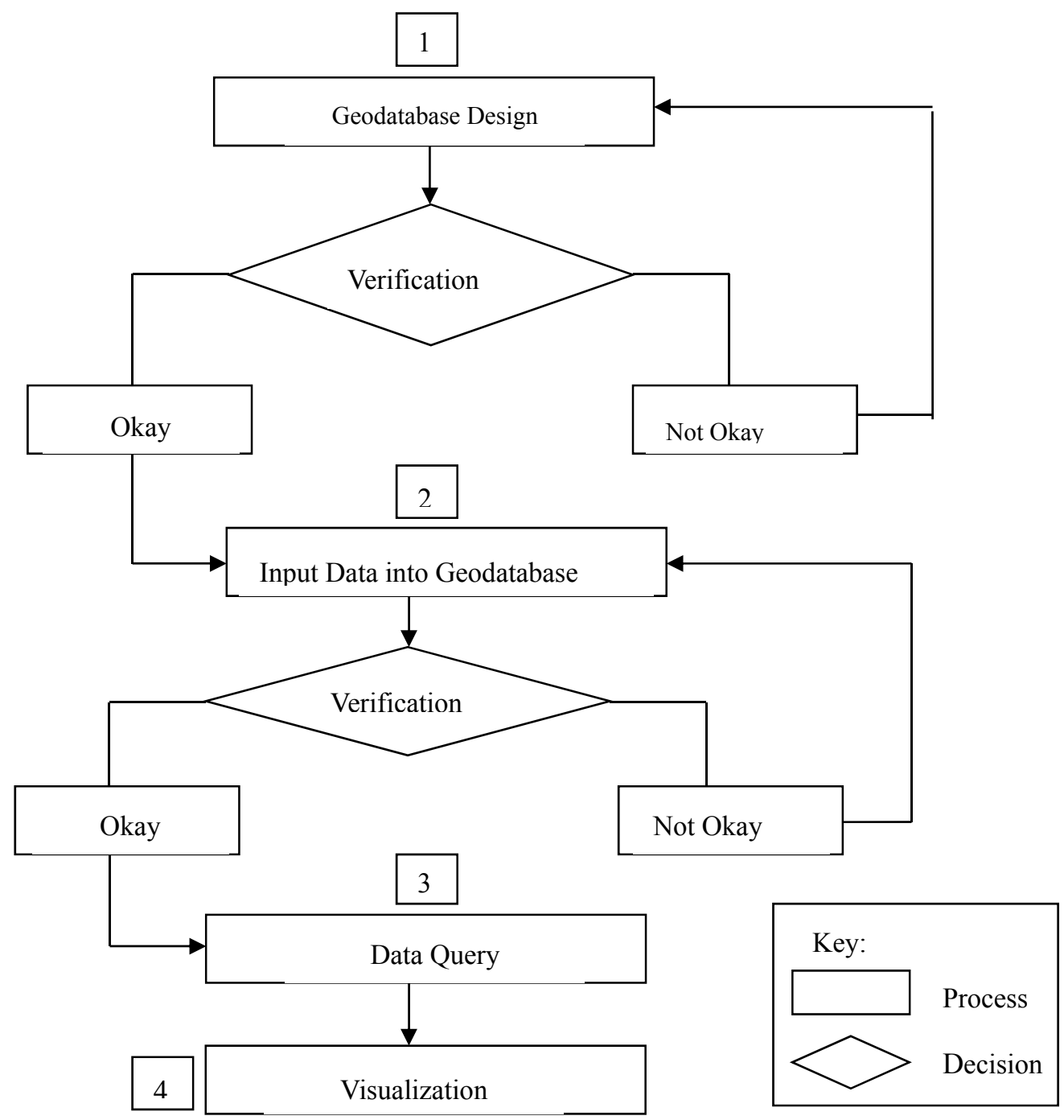

Figure 2. Work flow of DCCHGIS

\section{Results and Discussion}

\subsection{Implementation of the Geodatabase Design}

The Geodatabase was designed in the way that it allows flexibility and simplicity in managing and visualizing spatial and non-spatial information. Arc GIS Map Version 10.2.2 has been used to create the Geodatabase by using a file Geodatabase platform because it provides an opportunity to store large volume of data about 1TB.

Five fundamental elements of the Geodatabase were created to implement the DCCHGIS design (see figure 3). These include features classes, tables, attachment and relational classes (see figure 5). The feature classes were created by converting shapefile of the artifacts and historical sites. Due to difference in the UTM zones among the study sites, features classes that belonged to the same UTM zone were put into the same feature Dataset. For the purpose of simplifying the conversion processing of shapefile to feature classes, the model was built in model builder of Arc Map (see figure 4) to allow the conversion process to be replicated when shapefiles needed to be converted into feature class. This was made possible by using iterator tool in the model builder.

Tables were created to store descriptive information of the artifacts and historical site in the geodatabse. To ensure that there no redundancy and dependency among tables, normalization was carried out prior to data storage. Each table was developed to store a single type of information such as address names or ownership of 
artifacts/historical site (see figure 5). Lastly, the tables were linked with feature classes by using relationship class. To achieve this, two important parameters were necessary to be defined before the relationship is created. In this regard, cardinality type and keys (primary and foreign keys) were defined in each relationship existed between feature class and table. Based on the data configuration, 1 to 1 and 1 to many cardinality were chosen because they meet the conditions in this project as shown in figure 6. However, relationship classes had limitation in final map production because table's information could not be visualized in symbology in Arc Map. This led to the use of join tool to join the data in the table and feature class for final Map production.

Furthermore, the project aimed to add value to the design of Geodatabase by expanding its functionality and exploring more options for interactive visualization of artifacts and historical site data. This led to the use of attachment which gives a privilege for any data to be added in the Geodatabase. In this case, images of artifacts and historical sites were added in the Geodatabase so as to allow the user of the system to have a visual impression of how the captured artifacts and historical site look like in their natural setting. To achieve it, the attachments option in Arc Map 10.2.2 created tables that stored images then created relationship classes to link images to corresponding feature class. Finally, the user could be in a position to view images by simply clicking on the feature of interest in the data view of the arc Map (see figure 7).

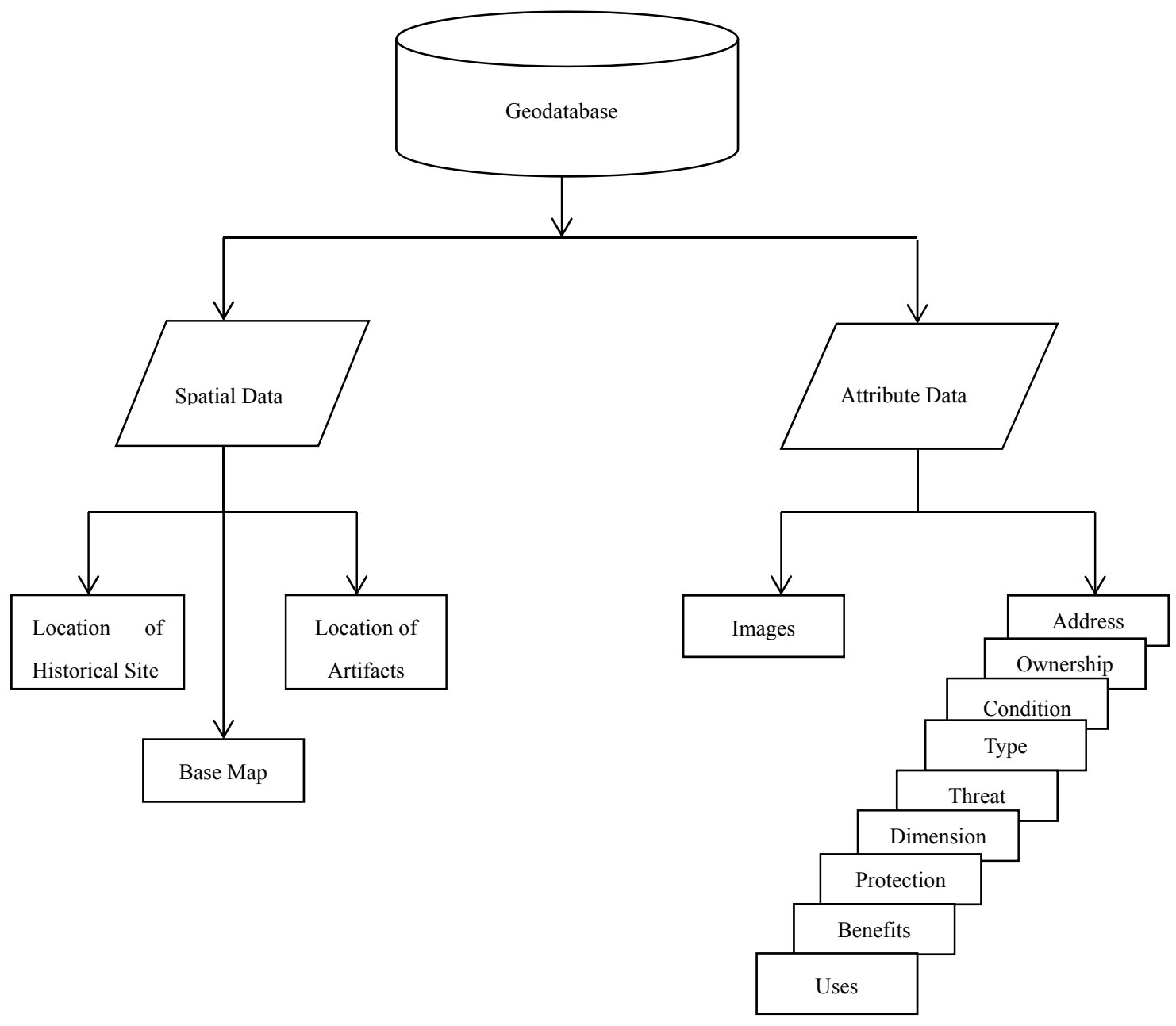

Figure 3. The design of DCCHGIS and its data 


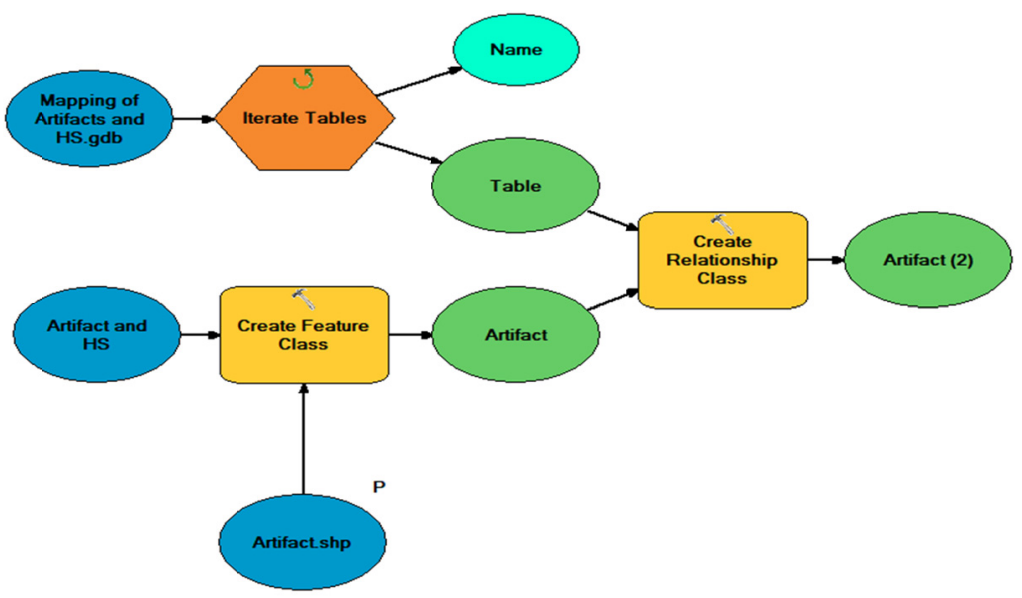

Figure 4. Model for converting shapefile to feature class and creating relationship classes as viewed in model builder in Arc Map

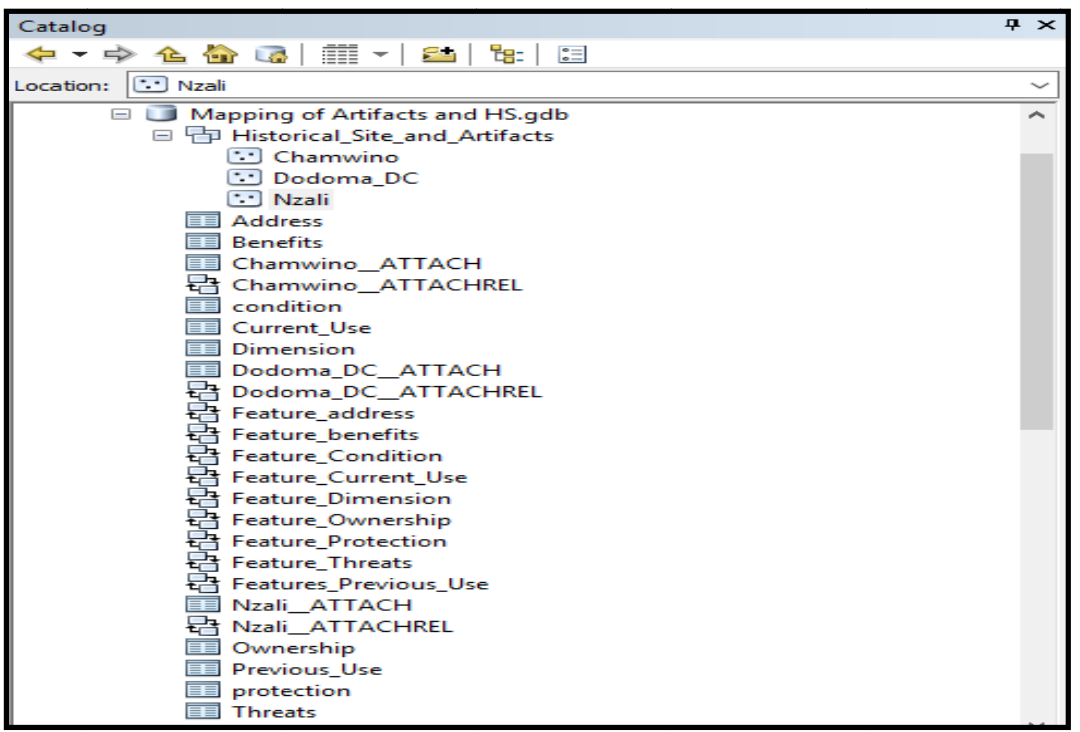

Figure 5. The contents of the DCCHGIS viewed in Arc Map Catalog

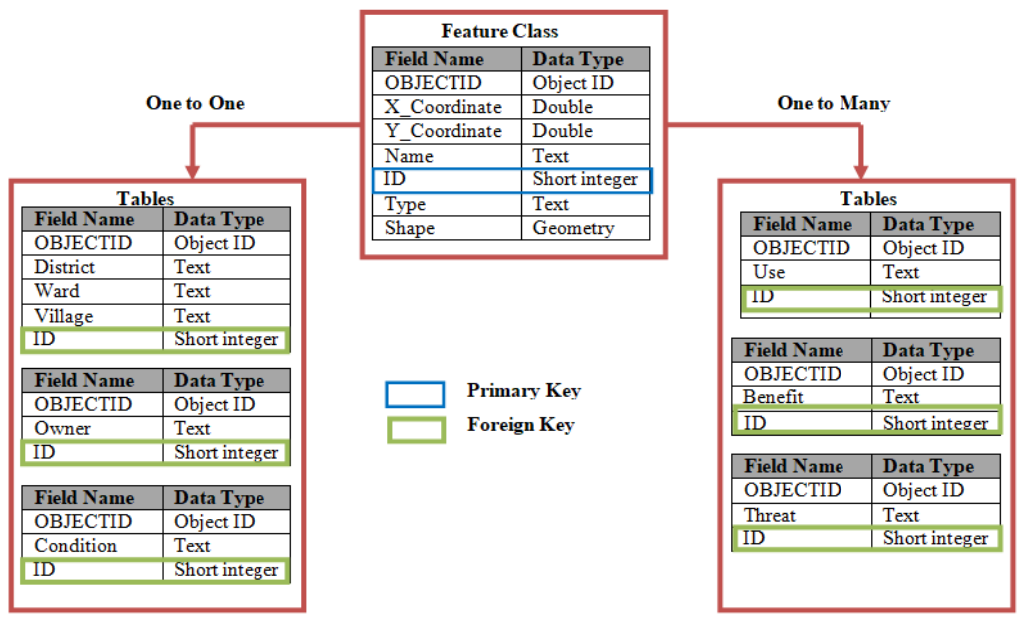

Figure 6. Example of Relationship between Feature class and tables in the Geodatabase of this project 
Upon completion, these layers were published to ArcGIS Online as a service in order to create a working interactive map layers. Publishing to ArcGIS Online via ArcGIS Desktop is the most accurate and accessible transfer of the data to a public forum (source?). The map layer was then edited to determine the web map's default scale, and orientation, as well as popup capabilities and legend. Once these settings were established, it was saved as a web map, which can be utilized by the DCCHGIS web application (Figure 3) to create a visualization of the entire database. This platform provides a means for the data contained within the archival collection to reach end users who may find the data important. This application allows for cartographic visualization of the material, basic spatial analysis using ArcGIS Online tools, and detailed searches using the search tool. ArcGIS Online was chosen due to its convenient connection with the ArcGIS Desktop with map (Figure 4) and Catalog as well as the fact that it is quickly evolving and adding new and useful tools, widgets and features to its platform.

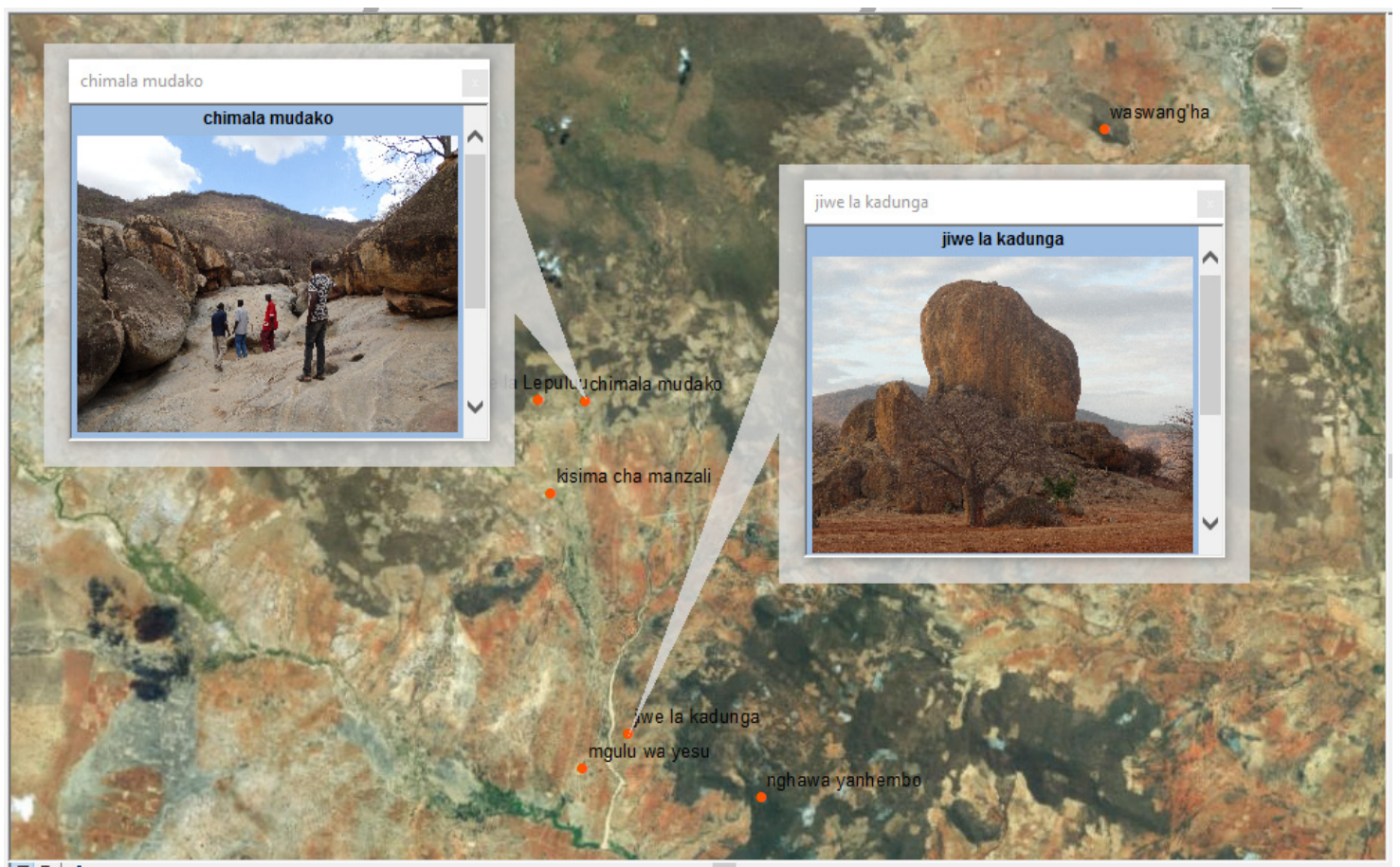

Figure 7. Data view in Arc Map showing pop-up images of historical site after clicking the feature

\subsection{Data storage in the Geodatabase}

The process of storing data in the Geodatabase was carefully done to ensure data are properly handled. The task was performed by one person to ensure uniformity in data storage. Before storing data, all collected data were inspected and reviewed by the project team to detect and correct any error in the data. After correcting errors, data coding was performed by giving ID to the artifacts and historical sites in order to properly enter them into the geodatabase. Attribute information of artifacts and historical site such as address name or dimension were entered manually into respective tables. Each table in the Geodatabase was developed to store only a single type of information for better data management. Finally, tables were linked with the feature classes using relationship class. The choice to keep individual table proved to promote data organization, security and system efficiency.

The captured $\mathrm{x}$ and $\mathrm{y}$ coordinates of artifact and historical site information were entered manually into Ms Excel for processing then imported into Arc Map to create shapefile. Then shapefile were converted into features by using the model in figure 4.

\subsection{Data Query and Visualization from the Geodatabase}

The aim of the project was to ensure that information stored in the Geodatabase is easily accessible in a simple and efficient manner. This led to creation of test queries and observes the results so as to judge the performance of the system. Queries are simply the normal use case scenarios, questions similar to what might be frequently asked the users of the system (burrows, 2016). In this regard, a number of queries were created and testes as shown in Table 3. Before queries were performed, feature class was linked with the tables of interest because attribute information were not stored in the feature class's table. The results of the queries proved that the system 
was efficient to search and retrieve information from the Geodatabase in a very efficient manner. Figure 8 shows query syntax and the result of query 1 whereby records that met the criterion were selected in the attribute table as seen in cyan colour.

Table 3. Sample queries

\begin{tabular}{ll}
\hline Queries & Attribute questions \\
\hline Query 1 & Which are artifacts or historical sites in good physical condition? \\
Query 2 & Which are artifacts or historical sites are used for rituals or worship? \\
Query 3 & Which are artifacts or historical sites are owned by the local community? \\
Query 4 & $\begin{array}{l}\text { What benefits do local communities get from the existence of artifacts or historical } \\
\text { sites? }\end{array}$ \\
\hline
\end{tabular}
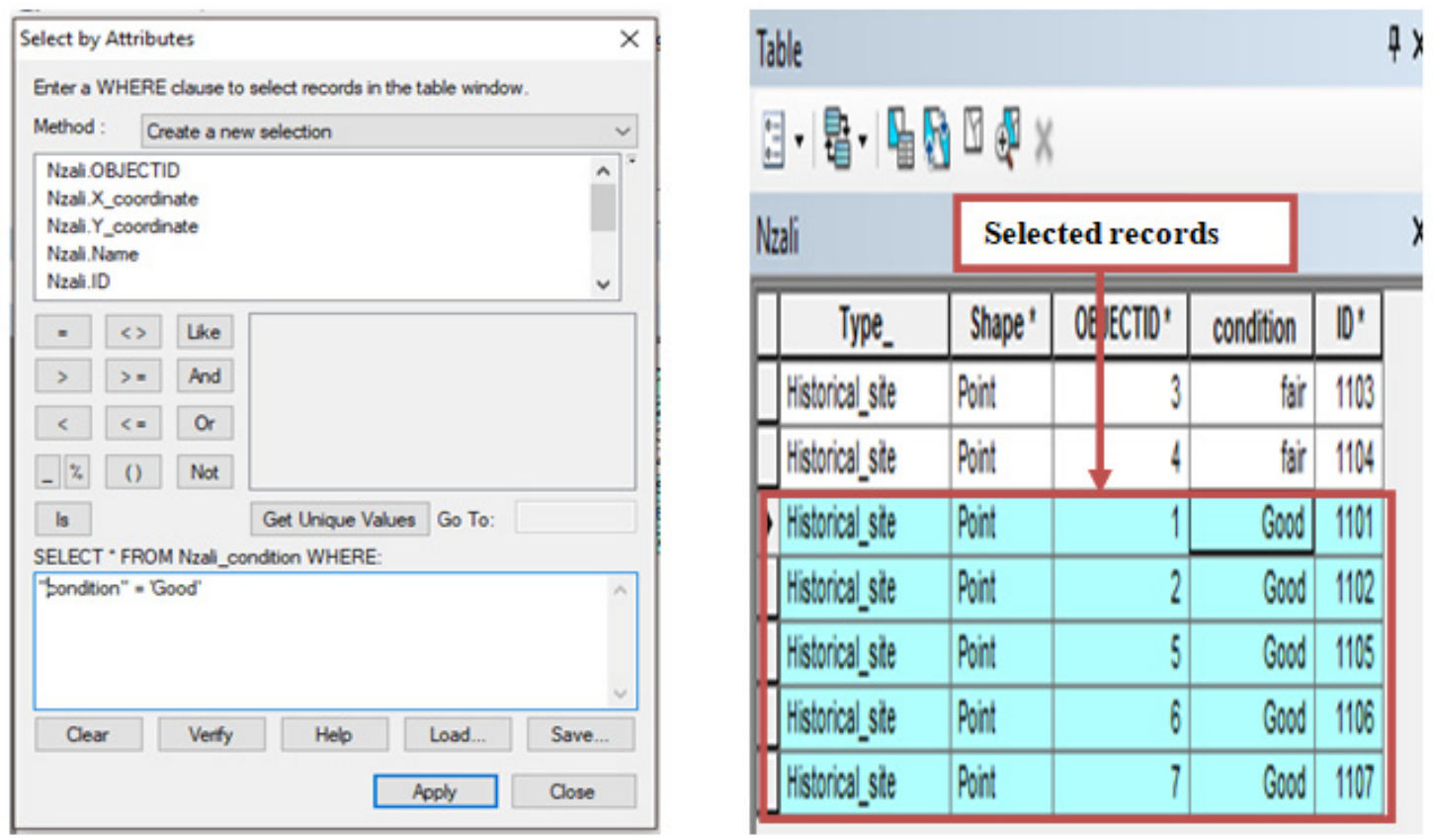

Figure 8. Sample Query 1 and the selected records in attribute table

In order to properly visualize data from Geodatabase, map layers (figure 9) were added in the table of content of Arc Map then followed by symbolization. Because features were geometrically represented as point in the vector format then symbolization was carefully performed to ensure that symbols selected have visible size and good colour. Also, features were labeled appropriately by using big font size.

The final maps were created for the purpose of sharing results with audiences at various levels from the grass root to national level. Maps produce were inspected and reviewed to ensure that they are of best quality and elaborative. Because of the scale effect and geographical extent of the study locations, a map for each study location was produced to allow good visualization of the artifact and historical site data at a large scale. Figure 10 shows a sample of a final map showing distribution of historical sites in Nzali village in Chamwino District. 


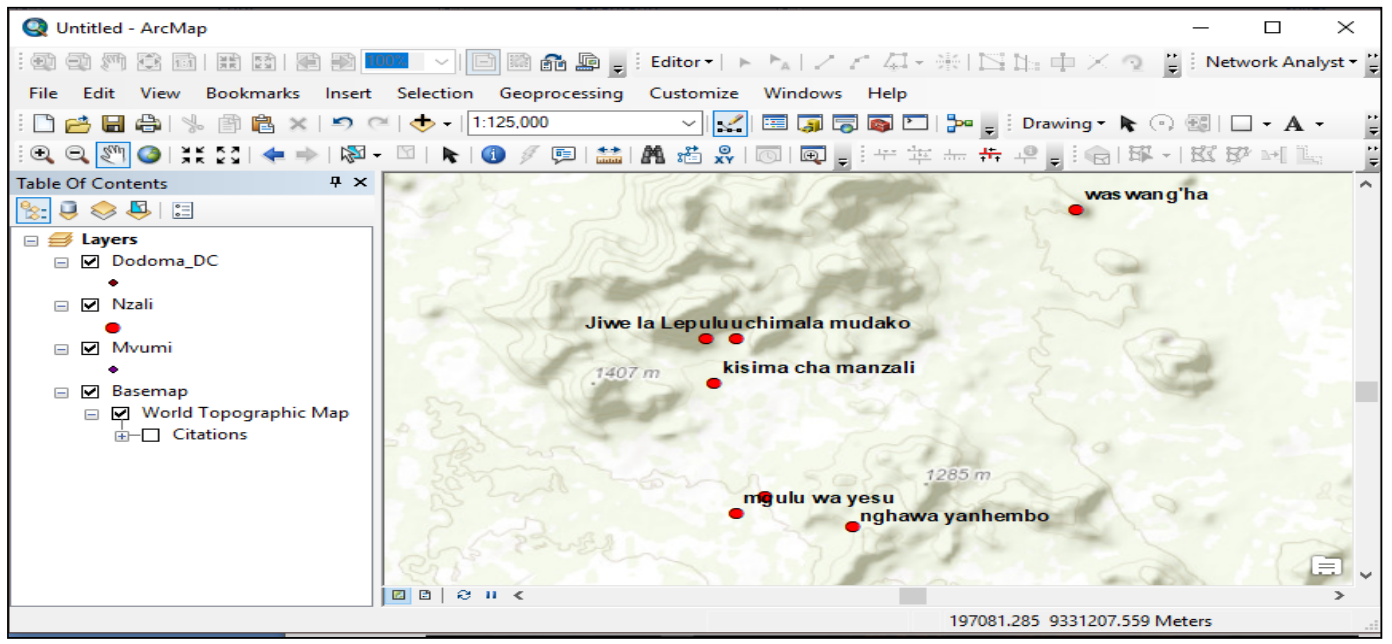

Figure 9. Sampled Historical Sites (red points) in Nzali Village overlaid on the Topographic Base map

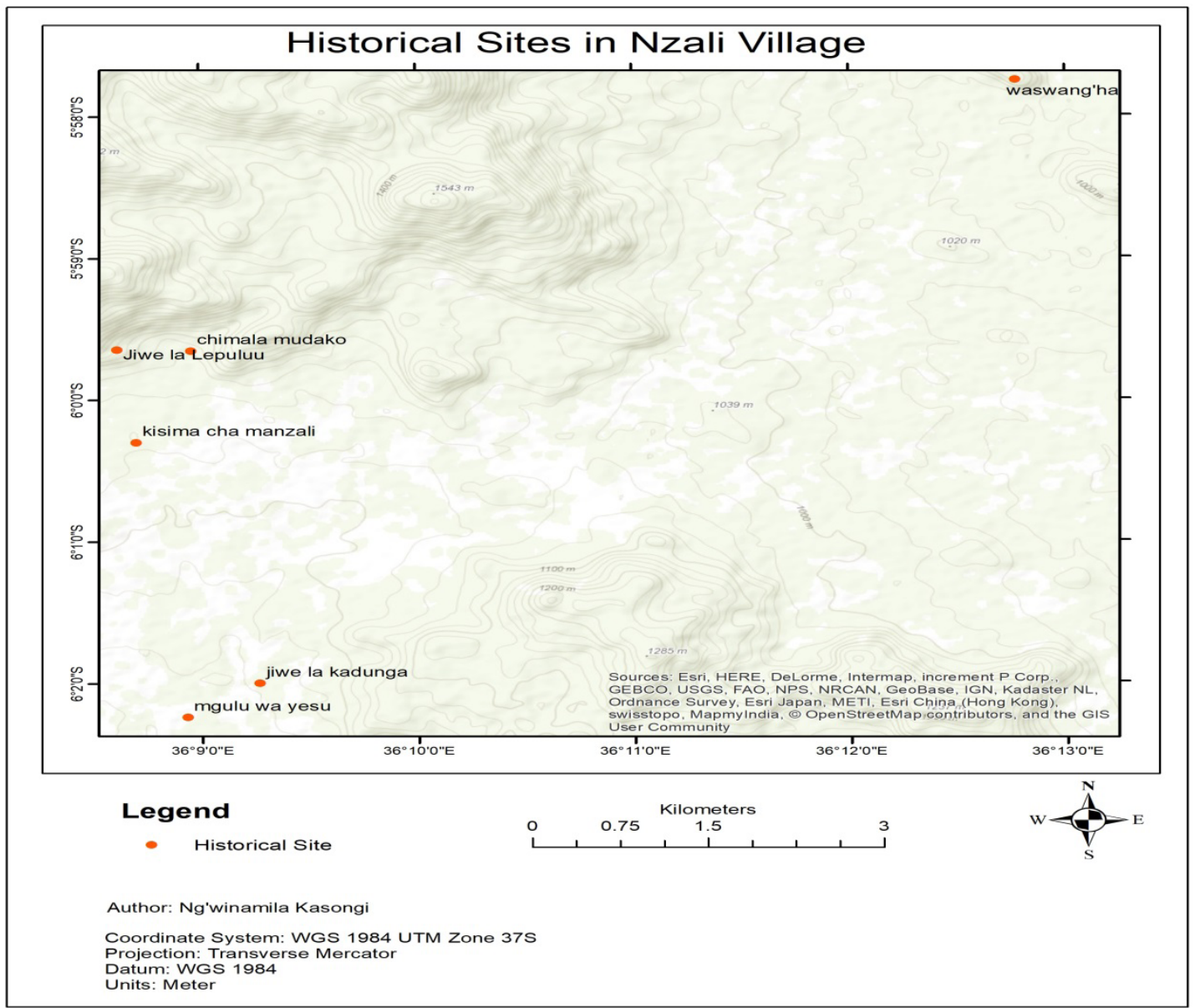

Figure 10. Final Map produced from Map layers shows distribution of the Historical Site in Nzali Village

\section{Conclusions and Recommendations}

This study has successfully combined techniques and technology used in GIS with the standards and cataloging processes of archival description of Dodoma Capital City Historical Geographic Information System (DCCHGIS). A web application that offers basic spatial analysis, allows for cartographic visualization of the data, and provides online public access to the artifacts image collection has been created. Although there are 
forerunners in terms of historical geographic information systems (HGIS) based on artifacts data and published to the web, none use established archival description guidelines, policies, and practices that could standardize the use of such data in a geodatabase. Using standardized policies, processes and techniques to describe and organize artifacts data used in creating geodatabases could greatly impact the future of historical and archival research and presentation.

Overall, the new geodatabase creation protocols used for the DCCHGIS study were successful. These protocols were effective due to the expansive nature of the available GIS tools and techniques used for both creating and organizing the artifacts data used in the HGIS geodatabase creation. The finished web application that was created on the DCCHGIS geodatabase provides useful cartographic visualization, spatial analysis, and online access to the archived artifacts data.

The methods and new geocoding protocols used during the creation of the DCCHGIS contribute to the GIS profession in several different ways. First, it highlights the ability of GIS to serve widely in support of other academic fields. Second, it demonstrates the value of library science or archival science and its description standards in the creation of geodatabases.

\section{Acknowledgement}

This study was funded by the University of Dodoma (UDOM), Tanzania. We are grateful for their support. We appreciate the assistance provided to us by the officials and the people involved in the study area. We thank the people in villages for accepting and participating in this study.

\section{References}

Burrows, C. (2016). Developing an Archaeological Specific Geodatabase to Chronicle Historical Perspective at Bethsaida, Israel. Masters Degree thesis, University of Southern California.

Chang, H. (2008). Autoethnography as Method. Walnut Creek, CA: Left Coast Press. 229pp. https://dx.doi.org/10.1177/1468794110379331

Chorley, R. J., \& Haggett, P. (Eds.). (2006). Models in Geography. Progress in Human Geography, 30(1), 107-113. https://dx.doi.org/10.1191/0309132506ph595xx

Genzuk, M. (2003). A Synthesis of Ethnographic Research. Occasional Papers Series. Center for Multilingual, Multicultural Research, Rossier School of Education, University of Southern California. Los Angeles. https://dx.doi.org/10.1080/14681369900

Heywood, I., Cornelius, S., \& Carver, S. (2006). An Introduction to Geographical Information Systems (3rd ed.). Pearson Education Ltd. Edinburgh UK.

Kessy, F., Mashindano, O., Rweyemamu, D., \& Charle, P. (2011). Poverty Escape Routes in Central Tanzania: Coping Strategies in Singida and Dodoma Regions. ESRF Discussion Paper No. 39, Vol. II. Retrieved from http//www.esrf.or.tz/docs/Discussion_Paper_39.pdf

Muganyizi, J. M. (2009). Land Use Changes within Agricultural Systems and their Implications on Food Security in Rungwe District, Tanzania. M.Sc Dissertation, University of Dar es Salaam, Dar es Salaam.

Reeve, D. E. (1996). Module 4: Attribute data. UNIGIS postgraduate diploma in GIS by distance learning. Course materials, $5^{\text {th }}$ edn. Manchester metropolitan university.

Shelley, F. M., \& Clarke, A. E. (1994). Human and Cultural Geography: A Global Perspective. Wm. C. Brown Communications Inc. Boulevard, USA.

United Republic of Tanzania. (1976). Capital Development Authority; National Capital City Master Plan, Dodoma.

United Republic of Tanzania. (1978). Capital Development Authority: Strategic Plan for the Development of National Capital City Dodoma: Background studies on issues affecting the implementation of the 1976 Master Plan.

United Republic of Tanzania. (2010). Environmental and Social Impact Assessment (ESIA). Final Report for Tanzania Strategic Cities Project Investment Sub-Projects. Dodoma Municipal Council and Capital Development Authority.

United Republic of Tanzania. (2015). Migration and Urbanization Monograph. National Bureau of Statistics. Retrieved from https://www.nbs.go.tz/nbs/takwimu/census2012/Migration_and_Urbanisation_Monograph.pdf 
Wenban-Smith, H. (2014). Urbanisation in Tanzania Population Growth, Internal Migration and Urbanisation in Tanzania 1967-2012: A Census-Based Regional Analysis. International Growth Centre (IGC). Retrieved from https://www.theigc.org/wp-content/uploads/2014/09/Wenban-Smith-2014-Working-Paper.pdf

\section{Copyrights}

Copyright for this article is retained by the author(s), with first publication rights granted to the journal.

This is an open-access article distributed under the terms and conditions of the Creative Commons Attribution license (http://creativecommons.org/licenses/by/4.0/). 